\title{
Integrating Materials and Manufacturing Innovation: a new forum for the exchange of information to integrate materials, manufacturing, and design engineering innovations
}

\author{
Charles H Ward
}

A number of contemporary studies have concluded that the research and engineering activities across materials, manufacturing, and product design need to be more closely coupled to enable responsive product innovation and delivery [1-3]. The recurrent themes in these studies point out that the fields of materials and manufacturing must strive to become more quantitative and predictive and have to reshape and integrate their engineering practices and outputs to better synchronize with product design engineering frameworks. This philosophy is strongly embraced by a relatively new discipline known as Integrated Computational Materials Engineering (ICME) which seeks to accelerate the development and deployment of advanced materials [3]. The complexities and opportunities of a fully engaged global research and manufacturing enterprise and the rapid speed of present day product design cycles demand a new ICME-based paradigm for how we work, collaborate, and share knowledge.

Hence the time is right to introduce Integrating Materials and Manufacturing Innovation (IMMI), a new journal that focuses on the issues and opportunities facing the materials and manufacturing community in building this new paradigm. The community is becoming ever more reliant on global collaboration, integrated design teams, digital data, computational modeling, and more complex and high throughput experimentation. In addition to integrating these themes in one publication venue, it is clear we need to evolve not only the way in which we disseminate ideas and information, but the format and richness in which it is shared. This journal will promote a discourse that fosters ICME, the accelerated implementation of advanced materials in the product design cycle, and the minimization of the disciplinary

Correspondence: Charles.Ward@wpafb.af.mil

Air Force Research Laboratory, Wright-Patterson AFB, OH 45433, USA seams between materials, manufacturing and design innovations. As dictated by the scope of the challenge, this dialogue will cover the entire materials life-cycle of discovery, development, design, manufacture, deployment, sustainment, and disposal and will engage both scientist and engineer alike.

Great strides have been made in developing experimental, computational and data management techniques across a number of technical disciplines to provide invaluable insight to solving scientific and engineering problems. Our community is entering an era where individual computational tools and dispersed experimental and modeling data must be brought together to create integrated toolsets that are made available to materials, manufacturing, and design engineers to create a Materials Innovation Infrastructure, as called for through the Materials Genome Initiative [4]. In fact, integration is at the very heart of IMMI; the tasks of integration across length and time scales as well as discipline boundaries are extraordinarily complex, but are at the forefront of engineering advances. Through its new format and topical focus, $I M M I$ will offer a novel forum to explore and advance this integration by presenting innovative approaches to overcoming the technical challenges in bringing experiment, models, and data together in order to provide advanced materials solutions faster and more efficiently than ever before.

The experimental component of research remains vitally important to progress in the digital age. Experiments are essential to informing models of material behavior, they provide new insight to stimulate the formulation of new theories, and they are absolutely indispensable to the validation of models for acceptance by the design engineer. The demands on experimental efforts are increased in an ICME-based paradigm, with experimental complexity (e.g. three-dimensional microstructural characterization) and high throughput 
capability pacing a Materials Innovation Infrastructure. $I M M I$ seeks new approaches to improve the quality and speed of generating critical experimental data.

The sibling to experiment is predictive modeling, which can enable a reduction in the large number of time-intensive and costly tests required in most product development cycles. The technical hurdles facing a materials innovation infrastructure are numerous, with many being associated with model maturity and the capture and linking of models through computational code. Given the complexity of the undertaking, it is exceptionally difficult to envision single, unified codes providing sources of integrated solutions, even in the midterm. Rather, we live in an environment where codes are and will be used in a modular fashion, likely having to interface with several other codes. Therefore, introduction of novel techniques that enable bridging length and/or time scale domains and approaches for representation of complex information, uncertainty quantification, model verification and validation, and seamless linking of models and data are all highly sought areas for contributions.

Currently, a unified approach to the critically important area of materials data management remains elusive and in great need of serious thought, discussion, and solutions. $I M M I$ aims to provide the venue where the community can discuss the critical elements of materials data to include ontology and database structure, capture, archiving, pedigree, standards and protocols for sharing, access and security, and materials informatics. As one example, the issues we face in both modeling and materials data suggest the solutions needed in the materials and manufacturing community may well be suited to burgeoning cloud computing concepts. Recognizing that access to data is a key barrier to progress, IMMI enables authors to publish the data and computational code files used in developing their manuscript, providing a citable reference for these elements of their work and direct access to data and code for the community.

Detailed case studies of engineering efforts to integrate experiment, modeling, and data to solve an enduring engineering problem in materials and manufacturing have been both instructive and inspirational to the community [5-9]. Thus, manuscripts that describe approaches taken in industry to truly integrate the materials, manufacturing, and product design engineering processes are also highly encouraged. Since IMMI is seeking to reach across traditional discipline lines to support an integrated scientific and engineering approach, it is hoped this venue will attract diverse input and perspectives to the journal.

$I M M I$ provides an open access platform for the presentation of original efforts. Open access means that all readers will have free access to articles appearing in the journal. To enhance the dissemination of information associated with temporal evolution in both modeling and experimental studies, IMMI will provide for the publication of videos along with the articles, in a similar manner to how one publishes figures in an article. In addition to the capability to host data, code, and video files with articles, we will also provide online commenting and discussion tools to promote the continued dialogue and deliberation of the ideas and results published. As the journal matures, we'll be looking to expand the types of digital information presented and how they are integrated into the journal article itself. Rather than bundling the articles into discrete monthly issues, they will be published on-line in a continuous fashion once they have passed peer review and final editing in order to make them available to the public as soon as possible. All articles published over a year's time will be assembled into an annual print version for purchase by individuals that desire a print copy.

Submissions to Integrating Materials and Manufacturing Innovation are welcomed via the journal's web site at www. immijournal.com. Suggestions for improvements to the journal as it grows are most welcome.

Received: 25 April 2012 Accepted: 11 June 2012

Published: 11 June 2012

\section{References}

1. Retooling Manufacturing: Bridging Design, Materials, and Production (2004) The National Academies Press, Washington, DC

2. Accelerating Technology Transition: Bridging the Valley of Death for Materials and Processes in Defense Systems (2004) The National Academies Press, Washington, DC

3. Integrated Computational Materials Engineering: A Transformational Discipline for Improved Competitiveness and National Security (2008) The National Academies Press, Washington, DC

4. (2011) Materials Genome Initiative for Global Competitiveness. Executive Office of the President, [http://www.whitehouse.gov/sites/default/files/ microsites/ostp/materials_genome_initiative-final.pdf]

5. Allison J, Li M, Wolverton C, Su XM (2006) Virtual aluminum castings: an industrial application of ICME. JOM J Miner Met Mater Soc 58:28-35. doi:10.1007/s11837-006-0224-4

6. Backman DG, Wei DY, Whitis DD, Buczek MB, Finnigan PM, Gao D (2006) ICME at GE: accelerating the insertion of new materials and processes. JOM J Miner Met Mater Soc 58:36-41. doi:10.1007/s11837-006-0225-3

7. Kuehmann CJ, Olson GB (2009) Computational materials design and engineering. Mater Sci Technol 25:472-478. doi:10.1179/174328408X371967

8. Chalupa R, Jiang L, Simka H, Shankar S, Thakurta D (2007) Virtual integrated processing for integrated circuit manufacturing. J Vac Sci Technol, A 25:1013-1018. doi:10.1116/1.2731341

9. Hirsch J, Karhausen KF (2011) History of ICME in the European Aluminum Industry. In: Allison J, Collins P, Spanos G (eds) 1st World Congress on Integrated Computational Materials Engineering. Wiley, Hoboken, NJ

doi:10.1186/2193-9772-1-1

Cite this article as: Ward $\mathrm{CH}$ : Integrating Materials and Manufacturing Innovation: a new forum for the exchange of information to integrate materials, manufacturing, and design engineering innovations. Integrating Materials and Manufacturing Innovation 2012 1:1. 